\title{
Glaube und Zweifel bei Sebastian Castellio und Johannes Calvin
}

Luca Baschera

Der aus Savoyen stammende Humanist Sebastian Castellio ${ }^{1}$ (Sébastien Castellion, 1515-1563) war zwischen 1541 und 1545 Leiter des "Collège de Rive" in Genf, zog aber in Folge einer Auseinandersetzung mit Johannes Calvin nach Basel. Dort publizierte er zahlreiche philologische Werke sowie zwei Übersetzungen der Bibel - eine französische und eine lateinische. Nach der Hinrichtung Michael Servets 1553 polemisierte er heftig gegen Calvin und Théodore de Bèze, die die Legitimität der Verfolgung von Häretikern durch den weltlichen Magistrat verteidigten. Vor allem durch den im Rahmen dieser Polemik anonym erschienenen Traktat De haereticis an sint persequendi sollte Castellio als "Apostel der Toleranz in die Geschichte eingehen. ${ }^{2}$

Im Folgenden wird das Augenmerk jedoch nicht auf die »toleranztheoretischen "Schriften, sondern vielmehr auf eine weitere Schrift Castellios gerichtet werden, die erst im 20. Jahrhundert erschien, nämlich De arte dubitandi et confidendi, ignorandi et sciendi. ${ }^{3}$ Castellios darin vorgelegte Theorie bezüglich des Verhältnisses von Glaube und Zweifel, Wissen und Unwissen soll in einem zweiten Schritt mit der Position des Reformators Johannes Calvin verglichen werden.

\footnotetext{
1 Zu Castellios Leben und Werk vgl. F. Buisson, Sébastien Castellion, sa vie et son œuvre (1515-1563), Paris 1892; H.M. Stückelberger, Calvin und Castellio, in: Zwingliana 7/2 (1939), 91-128.

2 Vgl. H.R. Guggisberg, Sebastian Castellio 1515-1563. Humanist und Verteidiger der religiösen Toleranz im konfessionellen Zeitalter, Göttingen 1997.

3 Die historisch-kritische Ausgabe dieses Werks wurde 1937 veröffentlicht und erschien 1981 in überarbeiteter Fassung, vgl. S. Castellio, De arte dubitandi et confidendi, ignorandi et sciendi [= DAD], hg. v. E. Feist Hirsch, Leiden 1981. Für eine ausführliche Analyse dieses Werks vor dem Hintergrund der Debatten im sechzehnten Jahrhundert vgl. St. Salvadori, Sebastiano Castellione e la ragione della tolleranza. L'ars dubitandi fra conoscenza umana e veritas divina, Mailand 2009.
} 


\section{Castellio: »Man glaubt doch immer nur an Zweifelhaftes!»}

Wie Castellio selbst zu Beginn des De arte dubitandi erklärt, hat er diese seine "Kunst des Zweifelns und des Glaubens, des NichtKennens und des Erkennens" entworfen, damit ein jeder "wie ein Felsen" mitten in "den Unruhen der [religiösen] Streitigkeiten" bestehen könne. ${ }^{4}$ Angesichts der extremen Vielfalt an theologischen Positionen sowie der Vehemenz, mit der diese von ihren jeweiligen Vertretern propagiert werden, möchte Castellio durch seine Schrift dem Leser zur Orientierung verhelfen. Castellio verspricht denjenigen, die sich seine "Kunst" aneignen, dass sie bewusst werden bestimmen können, wann geglaubt oder aber gezweifelt - im Sinne einer Urteilsenthaltung - werden soll, anstatt sich willkürlich der einen oder der anderen Partei anzuschließen.

Eine solche Bestimmung kann aber nur aufgrund von klaren Kriterien erfolgen, welche nicht nur "geglaubt«, sondern als wahr erkannt werden. Diese Kriterien bzw. »ersten Prinzipien« brauchten nicht bewiesen zu werden, denn ihre Evidenz sei unbestreitbar: Sie sind nämlich "sozusagen vom Finger Gottes in die Herzen aller eingeschrieben worden und können nicht getilgt werden $4 .{ }^{5}$ Nicht aufgrund einer speziellen Offenbarung, sondern auf ganz natürliche, unmittelbare Art und Weise kann der Mensch somit erkennen, dass es einen einzigen, die Welt regierenden und gerechten Gott gibt, und einsehen, wozu er moralisch verpflichtet ist (charitatis praecepta). Die Existenz Gottes und die moralische Pflicht des Menschen im Sinne des Liebesgebots werden ferner - so Castellio - durch die ratio erkannt, wobei diese keineswegs mit der autonomen Vernunft der Aufklärungsphilosophie zu verwechseln ist. Bei Castellio stellt die ratio vielmehr ein göttliches Prinzip dar, welches als »die Tochter Gottes" und »das ewige Sprechen [sermo] Gottes" definiert wird. ${ }^{6}$ Obschon jeder Mensch von Natur aus daran teilhat, partizipierte Jesus eminent an der göttlichen ratio, weshalb er im Johannesevangelium auch Logos - der griechische Begriff für sermo oder ratio - genannt wird. $^{7}$

An Gott braucht der Mensch also nicht zu glauben, weil die ratio dieses göttliche Prinzip, das zu seiner Kreatürlichkeit gehört - ihn erkennen lässt. Auf die Erkenntnis der Existenz Gottes und seines

\footnotetext{
DAD 1,1, S. 14,58-61.

5 DAD 1,20, S. 54,32-34.

6 DAD 1,25 , S. $65,40.44$

DAD 1,25, S. 65f,49-52.
} 
Willens (Liebesgebot) reduziert sich Castellio zufolge das, was der Mensch wissen soll, um ein gerechtes Leben zu führen und somit sein Ziel, sein »Heil« zu erlangen. Heilsnotwendig ist nicht der Glaube an eine geoffenbarte Wahrheit, sondern die natürliche Erkenntnis der ersten Prinzipien (deum esse et charitatis praecepta) durch die ratio.

Bereits aufgrund dieser wenigen Notizen wird deutlich, dass Castellios Ansatz grundsätzliche Aspekte nicht nur reformatorischer, sondern christlicher Theologie im Allgemeinen in Frage stellt.

(1) Im Bereich der Anthropologie lehnt er die christliche Sündenlehre explizit $a b$ und hält daran fest, dass "weder die Sinne noch der Intellekt des Menschen durch Adams Fall verdorben wurden ${ }^{8}{ }^{8}$ weshalb »das Urteil der Vernunft [d.h. der ratio im oben beschriebenen Sinn] « nach wie vor "richtig und besonnen ist «. Zwar muss festgestellt werden, dass die ratio sehr häufig von den Affekten benebelt und deshalb in der Ausübung ihrer Funktionen beeinträchtigt wird. Dies sei aber ein rein kontingenter, durchaus überwindbarer Zustand und nicht eine durch Adams Fall verursachte Grundbefindlichkeit des Menschen. Für Castellio ist folglich nur von einzelnen Verfehlungen, von Sünden im Plural zu sprechen, aber sicherlich nicht von der Sünde: Die menschliche ratio kann zwar erkranken, ist aber an sich nicht verdorben. ${ }^{10}$ Aus dieser pelagianischen Haltung heraus ist es nur konsequent, dass er das Sündenbekenntnis - ein zentraler Vollzug im reformierten Gottesdienst - für sinnlos hält und es in ein Bekenntnis der Güte und Gerechtigkeit des Menschen umwandelt: "Wir sind geneigt, das Gute zu tun, wir sind zu allem Guten nütze und gehorchen ununterbrochen den heiligen Geboten Gottes. «11

(2) Ebenso konsequent ist es, dass Castellio die Idee einer von auBen kommenden, den Menschen ansprechenden Offenbarung ablehnt.

\footnotetext{
8 DAD 1,27, S. 70,48f: "Quare sic statuimus, hominis sensum et intellectum non fuisse Adami peccato vitiatum."

9 DAD 1,26, S. 68,44f: "Quare hoc fatendum est rationis in homine iudicium esse rectum atque sanum."

${ }^{10}$ DAD 1,29, S. 72.

11 DAD 1,6, S. 27,60f: "Nos sumus ad bene faciendum proclives, ad omne bonum utiles et sine ulla intermissione sanctis dei praeceptis obediemus." Man beachte, dass Castellio fast wörtlich das Sündenbekenntnis aus La forme des chantz et prières ecclésiastiques (Genf, 1542) zitiert, dabei aber dessen Bedeutung bewusst umkehrt, vgl. Calvin Studienausgabe, Bd. 2: Gestalt und Ordnung der Kirche, hg. v. E. Busch et al., Neukirchen-Vluyn 1997, S.162,5-9: "Seigneur Dieu, Pere eternel et tout pouissant: nous confessons $[\ldots]$ que nous sommes $[\ldots]$ enclins à mal faire, inutiles à tout bien: et que de nostre vice, nous transgressons, sans fin et sans cesse, tes sainctz commandement."
} 
Wenn der Mensch allgemein im Stande ist, die ersten Prinzipien der Religion - d.h. das, was zu seinem »Heil notwendig ist - zu erkennen, braucht er selbstverständlich nicht, dass Gott ihm diese speziell offenbart.

(3) Die Ablehnung des traditionellen Sündenbegriffs bedingt auch grundlegende Veränderungen im Bereich der Soteriologie. Das Heil besteht für Castellio nicht mehr in der Erlösung von der Sünde durch die Vergebung Gottes aufgrund des Sühnetodes Christi (Röm 3,24-26), sondern in einer allmählichen Überwindung der durch den Einfluss der Affekte entstehenden "Seelenkrankheiten" (morbi animi). Diese gilt es durch eine mit dem Willen Gottes in Einklang stehende Lebensführung zu »heilen«. Das Heil ist somit kein Werk Gottes mehr, sondern ausschließlich Menschenwerk. ${ }^{12}$

(4) Schließlich bekommt auch die Christologie bei Castellio eine vollkommen neue Gestalt. Jesus - ob bei Castellio der Appellativ "Christus" immer noch eine Berechtigung hat, bleibt fraglich - ist nicht mehr der Fleisch gewordene Sohn Gottes, der auf Erden gesandt wird, um die Sünde der Welt zu tragen und den Menschen zu erlösen (Joh 1,14.29). Er ist vielmehr nur noch ein hervorragender Lehrer, der mit seinen Worten und Taten die Menschen unterwiesen hat, damit sie den Weg der Gerechtigkeit einschlagen und sich selbst von ihren Seelenkrankheiten befreien.

Ist Jesus in den Augen Castellios nichts anderes als ein Lehrer, so wird das Christentum - die Religion, die sich auf Jesus beruft - für ihn zu einer reinen "Gerechtigkeitslehre « (iusticiae doctrina) ${ }^{13}$ neben anderen, welche sowohl philosophischer als auch religiöser Natur sein können. Castellio zufolge erweist sich aber das Christentum als die "beste» (praestantissima) unter allen Gerechtigkeitslehren, weil es mehr als alle anderen vermag, »die Seelen [...] gerecht zu machen, und ihre Krankheiten am Besten heilt«. ${ }^{14}$ Diese seine These begründet Castellio durch zahlreiche Zitate aus dem Neuen Testament, ${ }^{15}$ wobei Neues und Altes Testament die "Heiligen Schriften" dar-

\footnotetext{
${ }^{12}$ Somit unterscheidet sich Castellio nicht nur von der reformatorischen, sondern auch von der römisch-katholischen Dogmatik, welche die Notwendigkeit der Gnade nie in Frage stellte, diese aber anders interpretierte als die reformatorische Theologie, nämlich als bloße gratia praeveniens, die dem Menschen die Wahl zwischen Glauben und Unglauben ermöglicht.Vgl. Konzil von Trient, Dekret über die Rechtfertigung, in: H. Denzinger, Enchiridion symbolorum, definitionum et declarationum de rebus fidei et morum, Freiburg i.Br. ${ }^{40} 2005$, Nr. 1525.

13 DAD 1,5 , S. 25,90f.

14 DAD 1,5, S. 23,44-46

15 DAD $1,5-7$, S. 23-28.
} 
stellten, in welchen die "christliche Lehre» vermittelt werde. ${ }^{16} \mathrm{Als}$ Grundtexte christlicher Gerechtigkeitslehre erfreuen sich diese Schriften besonderer Autorität. Da diese aber häufig in Frage gestellt wird, legt Castellio zahlreiche Argumente zu ihrer Bestätigung vor. ${ }^{17}$

Es ist auffallend, dass Castellio bisher weder von Glaube noch von Zweifel gesprochen hat. Die christliche Lehre - verstanden hier als Sammlung von Anweisungen zu einer tugendhaften Lebensführung - braucht nicht geglaubt zu werden, weil ihr Vorrang evident wird, sobald die ratio das Christentum im Lichte der - von ihr nicht geglaubten, sondern erkannten - "ersten Prinzipien" mit anderen Gerechtigkeitslehren vergleicht. Die Frage danach, was geglaubt werden soll, ist deshalb nicht dem Christentum als solchem inhärent, sondern stellt sich erst angesichts der Streitigkeiten bezüglich der korrekten Interpretation der heiligen Schriften. Da die "Schriftgelehrten" (sacrarum literarum doctores) verschiedene, häufig einander widersprechende Meinungen vertreten, möchte Castellio seinen Lesern eine Orientierungshilfe liefern, anhand deren sie bestimmen können, woran geglaubt werden soll. ${ }^{18}$

Gegenstand des Glaubens sind laut Castellio immer die "zweifelhaften Dinge" (dubia), welche nur auf Vermutungen (coniecturae) beruhen, weil sie sich der klaren Erkenntnis durch die Sinne oder den Intellekt entziehen. ${ }^{19} \mathrm{Da}$ in Bezug auf das Zweifelhafte ferner weder ein endgültiger Beweis noch eine endgültige Widerlegung geliefert werden kann, liegt es auf der Hand, dass darüber unendlich disputiert wird. Gerade die zweifelhaften Dinge können nun aber Gegenstand des Glaubens werden, denn: „Glauben bedeutet, Aussagen, die sowohl wahr als auch falsch sein könnten, Glauben zu schenken. ${ }^{20}$ Zum Zweifelhaften kann sich der Mensch folglich auf zwei unterschiedliche Weisen verhalten: Entweder enthält er sich eines Urteils oder er entscheidet sich, an eine Aussage zu glauben, bei

\footnotetext{
${ }^{16} \mathrm{DAD} 1,7, \mathrm{~S} .28,7 \mathrm{f}$.

17 DAD $1,8-16$, S. 29-47.

18 DAD 1,17, S. 48,25-31.38-40

${ }^{19}$ DAD 1,19, S. 51,3-5: "Dubia sunt ea, quae in coniecturis posita sunt, hoc est quae habent propabiblitatem, qualia sunt quae nec sensibus nec intellectu percipi possunt."

${ }^{20}$ DAD 1,19, S. 52,40f.: "Est igitur credere dictis seu veris seu falsis fidem habere." Castellio kennt die Unterscheidung zwischen belief und faith als solche nicht; bei der Lektüre seiner Texte entsteht dennoch häufig der Eindruck, dass für ihn faith letztlich in belief aufgeht.Vgl. I. Backus, The Issue of Reformation Scepticism Revisited. What Erasmus and Castellio Did or Did not Know, in: Renaissance Scepticisms, hg. v. G. Paganini und J.R. Maia Neto, Dordrecht 2009, S. 64-89, hier $80 f$.
} 
der er aber genau weiß, dass sie falsch sein könnte. Der Zweifel ist somit bei Castellio für den Akt des Glaubens selbst konstitutiv: Der Mensch glaubt gerade deshalb, weil er weiß, dass der Gegenstand seines Glaubens zweifelhaft ist.

Dass der Mensch laut Castellio jeweils selbständig bestimmt, was er glauben und was er anzweifeln wird, bedeutet ferner einerseits, dass der Glaube für ihn nichts anderes als ein Willensakt ist. ${ }^{21}$ Der Glaube ist keine Gabe Gottes, keine »Aneignungsursache» des Heils, sondern das Resultat einer bewussten Entscheidung des Menschen, der das Risiko auf sich nimmt, etwas Glauben zu schenken, was auch falsch sein könnte. Andererseits unterscheidet sich der Glaube grundsätzlich vom Wissen. Erkannt wird nur die Wahrheit und diese definiert sich als etwas, was keinen Raum für Zweifel zulässt. Da aber der Glaube gerade das Zweifelhafte als seinen Gegenstand hat, kann er keineswegs als eine Form von Wissen definiert werden. Vielmehr wird man sagen müssen - so Castellio -, dass der Glaube eine Art Vorstufe des Wissens darstellt. Wenn etwas erkannt wird, wäre es absurd, daran noch zu glauben, denn Glaube und Erkenntnis haben zwei verschiedene Geltungsbereiche: Glaube bezieht sich auf das Zweifelhafte, Erkenntnis hingegen auf das Gewisse und Wahre. ${ }^{22}$ Somit hebt die Erkenntnis den Glauben auf: "Wo das Wissen beginnt, hört der Glaube auf. ${ }^{23}$ Da aber viele, vor allem in Bezug auf die Interpretation der heiligen Schriften aufgeworfene Fragen die Erkenntnismöglichkeiten der Sinne und des Intellekts übersteigen, müssen sie auf Erden unbeantwortet bleiben. Man kann hoffen, dass beim Einbrechen des Reiches jeder noch so kleine Rest an Zweifel in reine Erkenntnis aufgehoben werden wird; diese eschatologische Hoffnung auf Erden realisieren zu wollen, wäre aber vermessen.

Wann ist es aber angemessen, etwas Zweifelhaftem doch Glauben zu schenken? Welche Kriterien sollen die Entscheidung, an etwas zu glauben, lenken? Ein erstes von Castellio benanntes Kriterium bezieht sich auf den Klarheitsgrad, mit dem bestimmte Dinge, die weder durch die Sinne noch durch den Intellekt begreifbar sind (supra sensus supraque intellectum), in der Schrift bezeugt werden. Somit wäre Zweifelhaftes "zu glauben, wenn es offensichtlich, oder anzuzweifeln, wenn es zweideutig, oder außer Acht zu lassen, wenn es

${ }^{21}$ DAD 1,19, S. 51f; 2,3, S. 89 f.

${ }_{22}$ Dabei ist wichtig festzuhalten, dass für Castellio die Erkenntnis sich zwar in Aussagen äußert, aber die Dinge selbst als ihren Gegenstand hat; der Glaube hat hingegen Aussagen als seinen Gegenstand, die sich auf Dinge beziehen, welche jenseits der Grenzen unseres Erkenntnisvermögens liegen.

${ }^{23}$ DAD 1,19, S. 52,47: "[...] ubi scientia incipit, ibi fides desinit." 
nicht mal zweideutig bei den heiligen Autoren vorliegt«. ${ }^{24}$ Höchstes Kriterium zur Bestimmung dessen, woran man mit guten Gründen glauben kann, ist aber die ratio selbst.

Der Glaube bezieht sich zwar auf den Inhalt biblischer Schriften, aber der Mensch sollte nur an das glauben, was der ratio plausibel erscheint. Der ratio - als »ewiger Tochter Gottes" - steht ferner zu, nicht nur die Schrift überall anzuzweifeln, wo ihre Botschaft mit den »ersten Prinzipien" nicht übereinstimmt, sondern sie manchmal auch zu korrigieren:

"Die ratio ist jene Erforscherin, Entdeckerin, Interpretin der Wahrheit, die, wenn etwas sowohl in den profanen als auch in den heiligen Schriften unklar oder durch die Zeit korrumpiert ist, es solange korrigiert oder anzweifelt, bis die Wahrheit selbst hervorleuchten wird. ${ }^{25}$

Die ratio übt somit zunächst eine negative Funktion aus, indem sie alles für unglaubwürdig erklärt, was den von ihr erkannten »ersten Prinzipien« sowie den Sinnen widerspricht. Im Bereich dessen, was weder vernunft- noch sinnes-widrig noch gewiss erscheint - d.h.im Bereich der zweifelhaften Dinge (dubia) -, kann der Mensch hingegen frei entscheiden, was er glauben will, im Bewusstsein, dass es sich dabei um bloße Meinungen handelt.

Dies bedeutet letztlich, dass sämtliche Dogmen christlicher Theologie - allen voran das trinitarische ${ }^{26}$ - als Meinungen angesehen werden, deren Berechtigung nicht größer als diejenige der ihnen widersprechenden »Häresien « ist. In dieser Perspektive verliert die Unterscheidung zwischen Dogma und Häresie selbst an Bedeutung. Ein Gegensatz besteht nur noch zwischen dem Wissen um Gott und seinem Willen und den unzähligen Meinungen über Dinge, die sich dem Urteil durch die Sinne und dieVernunft entziehen. Besser wäre es deshalb, sich auf den vernünftigen Kern der christlichen Religion (deum esse et praecepta charitatis) zu konzentrieren und die Dispute bezüglich der Dreieinigkeit Gottes und der Beziehung zwischen den trinitarischen Personen als müßig und gar gefährlich außer Acht $\mathrm{zu}$ lassen. ${ }^{27}$

\footnotetext{
${ }^{24}$ DAD 1,24, S, 62,14-17.

${ }^{25}$ DAD 1,25, S. 67,87-90: „Denique haec ratio illa est veritatis indagatrix, inventrix, interpres, quae, si quid in literis tum prophanis tum sacris vel obscurum vel tempore vitiatum est, aut corrigit aut in dubium tantisper vocat, donec tandem [...] veritas elucescat $[\ldots]$.

${ }^{26}$ Besprochen in DAD 2,2, S. 85-89.

${ }^{27}$ DAD 2,2, S. 85,15f.
} 


\section{Calvin: Glaube als »feste und gewisse Erkenntnis « und die Zerrissenheit der glaubenden Existenz}

Castellio plädiert für eine dogmatische Skepsis, welche mit einem anthropologischen Vertrauen einhergeht und in diesem gründet. Die Dogmen können und sollen als zweifelhafte Meinungen betrachtet werden, weil der Mensch im Stande ist, durch seine gesunde ratio universal geltende Prinzipien zu erkennen, im Lichte deren die sogenannten Dogmen sich als bloß partikulare Ansichten entpuppen. Bei Calvin begegnet hingegen ein dogmatischesVertrauen, gekoppelt mit einer grundsätzlichen anthropologischen Skepsis.

Zur Weisheit, nämlich zur wahren Erkenntnis Gottes und seiner selbst, ${ }^{28}$ kann der Mensch Calvin zufolge nur aufgrund einer Offenbarung gelangen. Zwar tut sich Gott auch in der Schöpfung kund, was theoretisch zu einer natürlichen Erkenntnis Gottes führen könnte. Unser Verstand ist aber durch einen »ungeheuren Schlamm von Irrtümern" derart bedeckt, dass er von sich aus nicht Gott er-kennen, sondern nur Götzenbilder erzeugen kann. ${ }^{29}$ Obwohl die "unsichtbare Gottheit" in der Schöpfung zur Schau gestellt wird, fehlen uns dennoch »die Augen, sie zu sehen, wenn wir nicht durch Gottes innere Offenbarung erleuchtet werden «. ${ }^{30}$ Im Gegensatz zu Castellio macht Calvin die Gotteserkenntnis von der Offenbarung gänzlich abhängig. Sogar die Erkenntnis Gottes im Spiegel der Schöpfung ist nur aufgrund einer vordergründigen, direkten, »inneren« Offenbarung möglich, ohne die der Mensch nicht Gott, sondern nur "Götzen« erkennen würde. Gleichzeitig stellt die Offenbarung auch die Quelle einer weiteren - mit der wahren Gotteserkenntnis stets einhergehenden - Einsicht dar, nämlich jener ins menschliche Unvermögen, Gott "natürlich« zu erkennen. Denn die Heilige Schrift - welche Gottes Offenbarungswort vermittelt - bekundet beides zugleich: Gott als gnädigen Vater, der den Menschen heilt, und den Menschen als der Sünde verfallene und deshalb heilsbedürftige Kreatur. ${ }^{31}$

\footnotetext{
${ }^{28}$ Johannes Calvin, Institutio christianae religionis [= ICR] I,1,1. Alle Zitate sind folgender Ausgabe entnommen: Johannes Calvin, Unterricht in der christlichen Religion - Institutio christianae religionis, übers. u. bearb. v. O. Weber, Neukirchen-Vluyn ${ }^{4} 1986$.

${ }^{29}$ ICR I, 5, 12

${ }^{30}$ ICR I,5,14 (meine Hervorhebung).

${ }^{31}$ ICR I,6,1f.
} 
Die Schrift vermittelt nun aber die Offenbarung, indem sie von demjenigen zeugt, in dem sich die Offenbarung vollkommen realisiert, dem Mensch gewordenen Gottessohn. In ihm hat Gott "jedwede Unterweisung dergestalt vollendet«, dass der Sohn "für das letzte und ewige Zeugnis von ihm [dem Vater] zu gelten hat «. ${ }^{32}$ In Jesus Christus - von Calvin auch "Gottes Weisheit im Fleische« genannt - werden nämlich beide Seiten der einen Wahrheit über Gott und Mensch endgültig geoffenbart. Gott erscheint »in der Gestalt seines eingeborenen Sohn als der Erlöser « ${ }^{33}$ der Mensch, dem in Christus Vergebung zugesprochen und dessen Natur durch den Geist erneuert wird, erscheint als Sünder. Calvin definiert die Sünde als jene

"erbliche Zerrüttung und Verderbnis unserer Natur, die in alle Teile der Seele hineingedrungen ist; diese macht uns zunächst vor Gottes Zorn zu Schuldigen, dann aber bringt sie auch in uns die Werke hervor, die die Schrift , Werke des Fleisches` [Gal $5,19]$ nennt.$^{34}$

Anders als bei Castellio - der nurvon "Sünden «als "Seelenkrankheiten" spricht - ist die Sünde für Calvin in erster Linie also ein Zustand, in dem sich der Mensch seit Adams Fall befindet: »Der ganze Mensch ist von Kopf bis zu Fuß wie von einer Sintflut derart über und über [mit Sünde] bedeckt, dass kein Teil unberührt ist, und deshalb wird alles, was von ihm kommt, als Sünde gerechnet. ${ }^{35}$ Deshalb kann der Mensch auf natürlichem Wege keine wahre Gotteserkenntnis erlangen, sondern bedarf einer externen Offenbarung, die ihn gleichzeitig sein Elend (die Sünde) und die Quelle seines Heils (Jesus Christus) erkennen lehrt.

Es wird nun ersichtlich, inwiefern sich bei Calvin die anthropologische Skepsis - die Überzeugung, dass der Mensch Sünder und deshalb zu keiner wahren Erkenntnis Gottes oder seiner selbst fähig sei - mit einem ausgesprochenen dogmatischen Vertrauen verbindet: Die Wahrheit, die durch die Schrift bezeugt und im christlichen Glaubensbekenntnis sowie in den Dogmen zum Ausdruck kommt, wird erkannt, nicht weil diese in den Herzen der Menschen "eingeschrieben" ist, sondern weil Gott durch seinen Geist das menschliche Herz erleuchtet und erneuert, damit dieses jene Wahrheit annehmen kann. Da der Mensch als gefallene Kreatur nicht mehr

\footnotetext{
32 ICR IV,8,7.

${ }^{33}$ ICR II,6,1.

${ }^{34}$ ICR II,1,8.

35 ICR II, 1,9 .
} 
im Stande ist, Gott zu erkennen, ist die Gotteserkenntnis bei Calvin immer eine Gabe. Gott wird von denjenigen erkannt, denen gegeben wird, die Offenbarung des Heils in Jesus Christus anzunehmen. Die wahre Gotteserkenntnis ist ein Aspekt des aus Gnade allein zugesprochenen Heils. Wer durch die Wirkung des Heiligen Geistes mit Christus geeint und somit der Gnade Christi teilhaftig wird, glaubt und erkennt im Glauben den wahren Gott, wie er sich in Christus geoffenbart hat. ${ }^{36}$

Der Glaube ist somit für Calvin »die feste und gewisse Erkenntnis des göttlichen Wohlwollens gegen uns, die sich auf die Wahrheit der in Christus uns dargebotenen Gnadenverheißung stützt und durch den Heiligen Geist unserem Verstand geoffenbart und in unserem Herzen versiegelt wird «. ${ }^{37}$ Zwei grundlegende Unterschiede zur Glaubensauffassung Castellios treten bei dieser Definition zu Tage: Erstens stellt der Glaube für Calvin eine Form von Erkenntnis und keinen bloßen Willensakt dar, weil sein Gegenstand nicht das Zweifelhafte, sondern die Wahrheit ist. Zweitens rührt der Glaube nicht von einer Entscheidung des Menschen her, sondern wird durch den Heiligen Geist erwirkt und ist somit eine Gabe.

Bedeutet nun aber die Bezeichnung des Glaubens als "feste" und "gewisse« Erkenntnis, dass sich Glaube und Zweifel für Calvin gegenseitig ausschließen? Calvin verneint dies dezidiert: "Wenn wir lehren, dass der Glaube gewiss und sicher sein soll, so verstehen wir darunter [...] nicht eine Gewissheit, die kein Zweifel mehr berührte [...]; nein, wir sagen, dass die Gläubigen immerfort im Kampfe liegen gegen ihren Mangel an Vertrauen. ॥ $^{38}$ Der glaubende Mensch ist somit nicht vom Zweifel befreit, sondern lebt vielmehr in einem Zustand innerer Zerrissenheit:

"[...] einerseits fühlt sich [das fromme Herz] in der Erkenntnis der göttlichen Güte mit Süßigkeit durchströmt, anderseits sieht es sich durch das Empfinden der eigenen Not bitter geängstigt, - einerseits ruht es sicher auf der Verheißung, die ihm das Evangelium zuteil werden lässt, anderseits erzittert es über dem Zeugnis der eigenen Ungerechtigkeit. " ${ }^{39}$

Dieser innere Kampf zwischen Vertrauen und Zweifel gründet Calvin zufolge in der bleibenden, auch nach erfolgter Erneuerung durch den Heiligen Geist weiterhin bestehenden Sündhaftigkeit

${ }^{36}$ ICR III, $1,3 \mathrm{f}$

${ }^{37}$ ICR III, 2,7.

${ }^{38}$ ICR III, 2,17 .

${ }^{39}$ ICR III,2,18. 
des Menschen. Zwar herrsche die Sünde im Christenmenschen nicht mehr, sie wohne aber immer noch in ihm, was wiederum der stete Kampf zwischen altem und neuem Menschen und die Notwendigkeit einer kontinuierlichen Buße bedinge. ${ }^{40}$

Insofern der glaubende Mensch ein Sünder bleibt, gehören - so Calvin - Zweifel und Glaube untrennbar zusammen. Gerade darin besteht nun aber der Unterschied zwischen seiner Position und derjenigen Castellios. Letzterer betrachtet den Zweifel als konstitutiv für den Glaubensakt sowie für den Glauben selbst: Gegenstand des Glaubens ist das Zweifelhafte und der Mensch, der sich dafür entscheidet, etwas Zweifelhaftem Glauben zu schenken, tut dies im Bewusstsein, dass der Gegenstand seines Glaubens falsch sein könnte. In diesem Sinne wird das Geglaubte Castellio zufolge auch immer zugleich angezweifelt. Bei Calvin ist der Zweifel hingegen konstitutiv für das glaubende Subjekt. Obwohl der Glaube aufgrund seines göttlichen Ursprungs eine "feste« und "gewisse " Erkenntnis darstellt, kann sich der Glaubende wegen seiner bleibenden Sündhaftigkeit von der schmerzlichen Erfahrung des Zweifels nicht befreien. Wegen seiner Sündhaftigkeit ist der Christenmensch sein ganzes Leben lang in einen inneren Kampf verstrickt, der die Zerrissenheit seiner Existenz als "gerechter und Sünder zugleich« bedingt. Wie das oben angesprochene dogmatische Vertrauen wurzelt also auch Calvins Überzeugung, dass der Zweifel unabdingbar zum Dasein des glaubenden Menschen gehört, in seiner anthropologischen Skepsis.

\section{Abschließende Bemerkungen}

Der Humanist Castellio ging von einer Verneinung der Ursünde und einem sehr positiven Menschenbild aus, bejahte die Möglichkeit einer natürlichen Gotteserkenntnis durch die ratio und gelangte zu einer Definition des Glaubens als vorsichtige Annahme zweifelhafter Inhalte im vollen Bewusstsein ihrer möglichen - und jedenfalls auf Erden nicht feststellbaren - Falschheit. Das wirksamste Mittel gegen die Anfechtungen, welche aus den endlosen Debatten über theologische Themata entstehen, ist laut Castellio der Zweifel selbst bzw. die gezielte Urteilsenthaltung. Zweifel und Glaube schließen sich bei ihm gegenseitig nicht aus, weil beide denselben Gegenstand haben: Man glaubt gerade an Zweifelhaftes, weshalb der Zweifel konstitutiv für den Glauben selbst ist.

\footnotetext{
${ }^{40}$ ICR III,3,10f.
} 
Der Reformator Calvin ging hingegen von einer Betonung der Notwendigkeit einer externen Offenbarung zur Erlangung wahrer Gottes- und Menschenerkenntnis aus, d.h. der Erkenntnis Christi als Erlösers sowie des Elends des in Christus erlösten Menschen. Eine solche Erkenntnis der Wahrheit der Offenbarung ist bei Calvin der Glaube, welcher Gott aus Gnade uns beschert. Da aber der glaubende Mensch Sünder bleibt, gehört die Erfahrung des Zweifels unvermeidlich zu seiner Existenz. Vom Zweifel kann sich der Mensch nicht befreien und insofern treten auch laut Calvin Glaube und Zweifel immer gemeinsam auf. Während bei Castellio aber der Zweifel Mittel gegen die Anfechtungen ist, stellt er bei Calvin die Anfechtung selbst dar. Als solche ist sie nicht zu überwinden; der Glaubende wird sich aber mit ihr auch nicht abfinden, sondern von ihr immer wieder dazu getrieben werden, Zuflucht bei Gott, dem Ursprung von Glauben und Hoffnung zu suchen: „Der in sich verwirrte Geist sammelt sich in Gott; in ihm wird er aufgerichtet, während er in sich selbst daniederliegt. " ${ }^{41}$

Sind Calvins und Castellios Positionen gegensätzlich, so schöpfen sie dennoch nicht alle Möglichkeiten der Verhältnisbestimmung von Glaube und Zweifel aus. Dies festzuhalten, ist umso wichtiger, als Castellios Ansichten häufig als skeptisch beschrieben ${ }^{42}$ und diejenigen Calvins als gar fanatisch angeprangert wurden. ${ }^{43}$ Castellio ist zwar skeptisch in Bezug auf das Dogma, vertritt aber eine Anthropologie, nach der der Mensch durchaus im Stande ist, die Wahrheit zu erkennen; im Gegensatz dazu würde ein Skeptiker auch diese Möglichkeit negieren und für eine sowohl dogmatische als auch anthropologische Skepsis plädieren. Analog dazu kann Calvin nicht als Fanatiker angesehen werden, weil er an der Sündhaftigkeit der menschlichen Natur auch nach erfolgter Erneuerung durch den Heiligen Geist festhält und deshalb den Zweifel als unüberwindbaren Bestandteil des Glaubenslebens betrachtet. Ein Fanatiker - oder Enthusiast - würde hingegen diese anthropologische Skepsis ablehnen und die Sünde sowie den Zweifel als durch die Wiedergeburt abgeschafft oder aber als durch menschliche Anstrengung überwindbar auffassen.

${ }^{41}$ ICR III, 2,23

${ }^{42} \mathrm{Vgl}$. R.H. Popkin, The History of Scepticism from Erasmus to Spinoza, Berkeley et al. 1979, 10f; Ders., Reason as the Rule of Faith in Castellio, the Early Socinians an the Jews, in: Aequitas, Aequalitas, Auctoritas. Raison Théorique et légitimation de l'autorité dans le XVI ${ }^{\mathrm{e}}$ siècle européen, hg. v. D. Letocha, Paris 1992, 195-203.

${ }^{43}$ Vgl. St. Zweig, Castellio gegen Calvin oder ein Gewissen gegen die Gewalt, Frankfurt a.M. 1987. 
Obwohl die Ansichten Castellios und Calvins deshalb keineswegs auf die Extreme des Skeptizismus einerseits und des Fanatismus andererseits reduziert werden können, spiegeln sich in ihnen doch zwei grundverschiedene Haltungen wider, die auch in der späteren Geschichte protestantischer Theologie immer wieder aufkommen sollten. Einerseits tritt Calvin als Verteidiger der Objektivität der Offenbarung, als Vertreter einer Christologie, bei der Christus als inkarnierter Sohn Gottes und Erlöser aufgefasst wird, sowie als Verfechter einer ausgeprägten Hamartiologie auf. Andererseits stellt Castellio die Weichen sowohl für rationalistische als auch für spiritualistische Entwürfe, indem er die Objektivität der Offenbarung in Frage stellt und eine vernünftige - oder aber mystische Gotteserkenntnis abgesehen von Christus für möglich hält; darüber hinaus wird Jesus bei ihm zum bloßen Lehrer zur Unterweisung von Menschen, die zwar noch nicht vollkommen, aber sicherlich nicht verdorben sind.

- Dr. Luca Baschera ist wissenschaftlicher Mitarbeiter am Institut für Schweizerische Reformationsgeschichte und Assistent für Praktische Theologie an der Universität Zürich. 